\title{
Small heterodimer partner as a predictor of neoadjuvant radiochemotherapy response and survival in patients with rectal cancer: A preliminary study
}

\author{
SUP KIM ${ }^{1}$, MINA JOO ${ }^{2}$, MIN-KYUNG YEO ${ }^{2}$, MOON-JUNE CHO ${ }^{1}$, \\ JUN-SANG KIM ${ }^{1}$, EUN-KYEONG JO ${ }^{3,4}$ and JIN-MAN KIM ${ }^{2,4}$ \\ Departments of ${ }^{1}$ Radiation Oncology, ${ }^{2}$ Pathology and Medical Science and ${ }^{3}$ Microbiology and Medical Science, \\ College of Medicine, Chungnam National University; ${ }^{4}$ Infection Control Convergence Research Center, \\ College of Medicine, Chungnam National University, Daejeon 35015, Republic of Korea
}

Received March 31, 2020; Accepted June 16, 2021

DOI: 10.3892/ol.2021.12969

\begin{abstract}
Small heterodimer partner (SHP) plays an essential role in the regulation of innate immune and inflammatory responses. The aim of the present study was to identify whether SHP levels are associated with cancer immunology and treatment outcomes in rectal cancer. SHP expression was analyzed via gene set enrichment analysis and the OncoLnc database. In addition, immunohistochemistry and reverse transcription-quantitative PCR analyses were performed on the tissues of patients with locally advanced rectal cancer, and the associations of SHP expression with the clinicopathological and hematological features or treatment response to preoperative radiochemotherapy (pRCT) were analyzed retrospectively. Furthermore, the present study investigated whether SHP expression correlated with immune infiltration levels and immune checkpoint molecules in rectal cancer. The results revealed that low SHP mRNA expression was significantly associated with an inflammatory response and poor prognosis. The nuclear expression of SHP was associated with clinical $\mathrm{N}$ stage, neutrophil count, lymphocyte count, neutrophil-lymphocyte ratio and complete pathologic response following pRCT. The low nuclear expression of SHP was associated with poor overall and distant metastasis-free survival (DMFS). In multivariate analysis, the low nuclear
\end{abstract}

Correspondence to: Professor Jin-Man Kim, Department of Pathology and Medical Science, College of Medicine, Chungnam National University, 266 Munwha-Ro Jung, Daejeon 35015, Republic of Korea

E-mail: jinmank@cnu.ac.kr

Professor Eun-Kyeong Jo, Department of Microbiology and Medical Science, College of Medicine, Chungnam National University, 266 Munwha-Ro Jung, Daejeon 35015, Republic of Korea

E-mail: hayoungj@cnu.ac.kr

Key words: rectal cancer, small heterodimer partner, cancer-related inflammation, radiochemotherapy, prognosis expression of SHP was identified as a significant independent prognostic factor for DMFS and a marginally significant prognostic factor for overall survival in rectal cancer. Furthermore, patients with low SHP expression exhibited higher neutrophil and $\mathrm{CD} 8^{+} \mathrm{T}$ cell infiltration levels and higher PD-L1 expression in rectal adenocarcinoma. These results indicate that SHP may act as an anti-inflammatory mediator via the regulation of systemic and local immune responses in rectal cancer. Moreover, SHP might be useful a potential marker or therapeutic target in rectal cancer.

\section{Introduction}

The prognosis of locally advanced rectal cancer (LARC) depends largely on the tumor stage at diagnosis. In the preoperative setting, the post-treatment tumor, node and metastasis (TNM) stage and pathological complete response to preoperative radiochemotherapy (pRCT) have been reported to be associated with disease-free survival (1). However, these factors cannot be determined prior to surgery. Predictors of tumor response and long-term outcome that are identifiable before surgery are necessary for designing a customized management plan for each patient to increase the survival rate. Therefore, it is important to identify additional biomarkers to predict and select patients who will respond favorably to treatment. In addition to improving outcomes, studies on biomarkers may also provide novel insights into the molecular mechanism of LARC.

Small heterodimer partner (SHP; also known as NR0B2) is an orphan member of the nuclear receptor superfamily that contains a putative ligand-binding domain (LBD), without the classical DNA-binding domain $(2,3)$. SHP has been shown to bind to specific activating molecules through LBDs and interact with other coactivators and corepressors to mediate transcriptional regulation. Due to this ability, dysfunctional SHP signaling leads to a wide variety of metabolic, reproductive and proliferative disorders $(4,5)$. The role of SHP has been extensively studied in liver and breast cancer (6). In liver cancer, previous studies have reported that SHP exhibits potent tumor suppressive activity by inhibiting cellular growth 
and increasing the sensitivity of tumor cells to apoptotic stimuli (7-9). In addition, a study of $\mathrm{SHP}^{-/}$mice suggested that SHP plays a critical role in tumor suppression by repressing the transcription of cell proliferation-associated genes (8). Furthermore, another mechanism of SHP has been indicated to be the inhibition of estrogen-related receptor $\gamma$, resulting in the interruption of Notch3 signaling through the activation of miR-206 (10). In breast cancer, it has been consistently reported that there are close associations between SHP and estrogen-related signaling (11-13). SHP blocks estrogen action by inhibiting estrogen receptor-mediated transcriptional activation and inducing peroxisome proliferator-activated receptor $\gamma$, which is an effective inhibitor of aromatase expression $(11,12,14,15)$. Induction of the farnesoid $X$ receptor-SHP-liver receptor homolog-1 pathway has been suggested as a potential new therapeutic approach for the repression of tumor growth and induction of apoptosis in breast cancer (16).

The role of inflammation in cancer is well established (17). Cancer-associated inflammation can increase the risk of cancer and impact the progression and treatment response of patients with various types of cancer, including colorectal, prostate and bladder cancer (17). A meta-analysis demonstrated that markers of the systemic inflammatory response, such as the C-reactive protein (CRP) level, neutrophil-lymphocyte ratio (NLR), lymphocyte-monocyte ratio (LMR) and platelet-lymphocyte ratio (PLR) could be useful in predicting treatment response and monitoring progression in patients with colorectal cancer (18-21). In addition, local inflammatory markers, such as the tumor-to-stroma ratio, Klintrup-Makinen score and Galon immunoscore can predict the prognosis of patients with colorectal cancer $(22,23)$. Therefore, there is a growing interest in biochemical mediators linking systemic and tumor inflammatory responses.

SHP has been shown to play an intricate role in the prevention of excessive inflammation by regulating the innate immune system (24-27). However, the role of SHP expression in cancer-associated inflammation and the clinical outcome of rectal cancer has not yet been investigated. In the present study, SHP expression and its association with systemic inflammatory markers, treatment response and survival in patients with rectal adenocarcinoma (READ) were investigated using a combination of bioinformatics and immunohistochemistry.

\section{Materials and methods}

Bioinformatics analysis. Gene Set Enrichment Analysis (GSEA) was performed as previously described (28). Briefly the mRNA-Seq profiles (illuminahiseq_rnaseqv2-RSEM genes_normalized) and clinical data of patients with READ were obtained from Firehose (https://gdac.broadinstitute. org/). The Cancer Genome Atlas (TCGA) RNA-Seq data were cross-referenced with the clinical information recorded for the patients. Patients with missing clinical data and/or expression values were excluded from further analyses. Data from 95 samples were included in the study. The mRNA-Seq data were normalized using the Rank Normalize module in GenePattern (http://broadinstitute.org/cancer/software/genepattern). To identify the best cutoff for inflammation by using GSEA, the patients were then ranked based on SHP expression and divided into high (30\%, ranking 1-29) and low (70\%, ranking 30-95) expression groups according to rank. Phenotype labels were permuted 1,000 times, and a normalized $\mathrm{P}<0.05$ and false discovery rate (FDR) of $<0.25$ were selected as statistically significant enrichments.

The OncoLnc database (http://www.oncolnc.org/) was used to determine whether the expression of SHP was associated with the overall survival (OS) of patients with READ. The SHP (NR0B2) gene was queried and a Kaplan plot for READ was generated.

Analysis of LARC datasets was carried out essentially as previously described (28). The SHP expression patterns were downloaded from the GSE15781 dataset for the comparison of pre-pRCT LARC and post-pRCT LARC patient groups (29). To compare the univariate differential expression in each dataset, RNA data were analyzed using the Wilcoxon-Mann-Whitney test.

Tumor Immune Estimation Resource (TIMER) is a resource for the systematic analysis of immune infiltrates for various types of cancer (https://cistrome.shinyapps.io/timer/) (30). TIMER applies a previously published statistical method of deconvolution to infer the abundance of tumor-infiltrating immune cells from gene expression profiles (30). The association of SHP expression with the abundance of various immune cells was analyzed using the TIMER database. In addition, the association between SHP expression and the gene expression of immune-suppressive molecules was investigated.

Patients and pretreatment evaluation. Between March 2003 and December 2011, 89 patients with LARC who had been treated with pRCT at the Chungnam National University Hospital (Daejeon, Republic of Korea) and for whom pre-treatment tissue blocks were available were retrospectively enrolled in the study. Eligibility for the study was determined based on the following criteria: i) Histological evidence of READ; ii) tumor extension through the bowel wall (T3-T4) or pelvic lymph node involvement without evidence of distant metastasis; and iii) presence of a resectable tumor. The exclusion criteria were as follows: i) Patients who had previous history of other cancers; ii) patients who had received previous curative resection for any colorectal tumor lesion; and iii) patients who had distant metastasis in the initial diagnosis. Patient and tumor characteristics are listed in Table I. Essential pre-treatment workups included a complete history, physical examination, complete blood count, serum chemistry, carcinoembryonic antigen (CEA) level analysis, chest radiography, abdominal/pelvic computed tomography (CT) and colonoscopy with biopsy. The TNM stage prior to clinical treatment was determined mainly by CT imaging. An informed consent form was signed by all patients. Biopsied tumor tissues were collected consecutively from patients at Chungnam National University Hospital between March 2003 and December 2011, and were analyzed following study approval from the institutional review board (IRB) of Chungnam National University Hospital (IRB no. 2017-07-037).

Radiotherapy treatment and evaluation of tumor response. Radiation was delivered via 6- and 10-MV photons using a three-field technique (posterior and bilateral fields) in most 
Table I. Association of SHP with clinicopathological features in patients with locally advanced rectal cancer.

\begin{tabular}{|c|c|c|c|c|}
\hline \multirow[b]{2}{*}{ Characteristics } & \multirow[b]{2}{*}{ Total, n (\%) } & \multicolumn{3}{|c|}{ SHP expression, n (\%) } \\
\hline & & Low $(n=52)$ & $\operatorname{High}(\mathrm{n}=37)$ & P-value \\
\hline Age, years & & & & 0.824 \\
\hline$<60$ & $57(64.0)$ & $34(65.4)$ & $23(62.2)$ & \\
\hline$\geq 60$ & $32(36.0)$ & $18(34.6)$ & $14(37.8)$ & \\
\hline Sex & & & & 0.809 \\
\hline Male & $65(73.0)$ & $37(71.2)$ & $28(75.7)$ & \\
\hline Female & $24(27.0)$ & $15(28.8)$ & $9(24.3)$ & \\
\hline Tumor distance from anal verge, $\mathrm{cm}$ & & & & 0.822 \\
\hline$<6$ & $58(65.2)$ & $33(63.5)$ & $25(67.6)$ & \\
\hline$\geq 6$ & $31(34.8)$ & $19(36.5)$ & $12(32.4)$ & \\
\hline CEA before RCT, ng/ml & & & & 0.822 \\
\hline$\leq 5$ & $58(65.2)$ & $33(63.5)$ & $25(67.6)$ & \\
\hline$>5$ & $31(34.8)$ & $19(36.5)$ & $12(32.4)$ & \\
\hline cT stage & & & & 0.224 \\
\hline $\mathrm{T} 2-3$ & $76(85.4)$ & $42(80.8)$ & 34 (91.9) & \\
\hline $\mathrm{T} 4$ & 13 (14.6) & $10(19.2)$ & $3(8.1)$ & \\
\hline cN stage & & & & 0.004 \\
\hline N (-) & $6(6.7)$ & $0(0.0)$ & $6(16.2)$ & \\
\hline $\mathrm{N}(+)$ & 83 (93.3) & $52(100.0)$ & $31(83.8)$ & \\
\hline
\end{tabular}

Data were analyzed using Fisher's exact test. SHP, small heterodimer partner; CEA, carcinoembryonic antigen; RCT, radiochemotherapy; cT, clinical T; cN, clinical N.

patients. Treatment was planned via computerized dosimetry, and a dose of $1.8 \mathrm{~Gy} /$ fraction was prescribed to cover the planning target volume. Radiotherapy was administered 5 days per week, once a day, at $1.8 \mathrm{~Gy} /$ fraction. Pelvic radiotherapy was performed with 45 Gy in 25 fractions over a period of 5 weeks, and followed by a booster dose of $5.4 \mathrm{~Gy}$, administered to the primary tumor in 3 fractions using 2 lateral fields. The clinical target volume contained the primary tumor, the mesorectum, the presacral space and lymph nodes, including the perirectal, presacral, internal iliac and/or external iliac nodes, as indicated. For the entire pelvic field, the superior border was located at the L5-S1 interspace, and the inferior border was located $3-4 \mathrm{~cm}$ below the primary tumor. The lateral border was located $1.5 \mathrm{~cm}$ outside the true bony pelvis. For the lateral fields, the posterior margin was $1.5 \mathrm{~cm}$ behind the anterior bony sacral margin, and the anterior border generally comprised the anterior acetabulum. Preoperative chemotherapy was administered concurrently with radiation therapy. Patients received oral chemotherapy consisting of 2 cycles of capecitabine and leucovorin, according to the chemotherapy protocol of Chungnam National University Hospital. At $\sim 6$ weeks after the completion of pRCT, the patients underwent definitive surgery. Surgical management included a sphincter-preservation approach, whenever possible, via the total mesorectal excision technique. Pathological evaluation of surgical specimens, including the primary tumor and resected nodes, was performed by a specialist pathologist. The pathological TNM staging, histological grading for
LARC and modified Ryan scheme for tumor regression score were determined at the time of surgical resection and were based on the 8th edition of the American Joint Committee on Cancer staging system (31). The complete absence of residual tumor cells in the primary tumor was defined as a pathologic complete response (pCR).

Immunohistochemistry (IHC). Rectal adenocarcinoma tissues were fixed with $10 \%$ buffered formalin for $24 \mathrm{~h}$ at room temperature. The expression of SHP was analyzed by the IHC of paraffin-embedded tissue sections from patients with LARC. Sections from paraffin blocks with a thickness of $3 \mu \mathrm{m}$ were used for IHC. Endogenous peroxidase blocking $(0.03 \%$ $\mathrm{H}_{2} \mathrm{O}_{2}$ ) was performed for $10 \mathrm{~min}$ at room temperature. A rabbit polyclonal antibody against NR0B2 (cat. no. ab186874; Abcam) was diluted at 1:400 with antibody diluent (DaKo antibody diluent, cat. no. S3022; Dako; Agilent Technologies, Inc.), and tissue sections were incubated in the mixture overnight at $4^{\circ} \mathrm{C}$ in a humid chamber then washed 3 times with $0.05 \%$ TBS-T. After washing, samples were incubated in $100 \mu 1$ secondary antibody [EnVision + Single Reagents horse-radish peroxidase (HRP); cat. no. K4003; Dako; Agilent Technologies, Inc.] for an additional $20 \mathrm{~min}$ at room temperature followed by additional washing. The reaction products were visualized after 5 min in diaminobenzidine plus a substrate-chromogen solution. The slides were counterstained with Meyer's hematoxylin for $30 \mathrm{sec}$ at room temperature and mounted. Careful rinses with several changes of PBS were performed between each stage 
of the procedure. Two experienced pathologists who had no access to clinical information examined and scored the slides by using a light microscope (BX51; Olympus Corporation). The nuclear staining of the tumor was divided into 4 grades based on staining intensity: Score 0, no staining; score 1, weak staining; score 2 , intermediate staining; score 3 , strong staining. In cases of heterogeneous staining within samples, the higher score was selected if $>50 \%$ of the cells had a higher staining intensity. Cases with a score of 0 or 1 were categorized into a low expression group, whereas those with a score of 2 and 3 were categorized into a high expression group.

RNA extraction from formalin-fixed paraffin-embedded (FFPE) samples and reverse transcription-quantitative PCR $(R T-q P C R)$. Total RNA was extracted from $10-\mu \mathrm{m}$ slices of FFPE samples from potentially eligible patients $(n=12)$ using a Qiagen RNeasy ${ }^{\circledR}$ FFPE kit (Qiagen, Inc.) following the manufacturer's protocol. RNA quantitation and purity were measured using a NanoDrop ${ }^{\mathrm{TM}}$ ND-8000 spectrophotometer (Thermo Fisher Scientific, Inc.).

cDNA was synthesized by RT using SuperScript ${ }^{\mathrm{TM}}$ II Reverse Transcriptase (cat. no. 18064; Invitrogen; Thermo Fisher Scientific, Inc.) according to the manufacturer's protocol. qPCRs were carried out using Rotor-Gene SYBR-Green PCR kits (cat. no. 204074; Qiagen, Inc.) in a Rotor-Gene Q 2plex system (cat. no. 9001620; Qiagen, Inc.). The samples were amplified for 40 cycles as follows: $95^{\circ} \mathrm{C}$ for $10 \mathrm{sec}$ and $60^{\circ} \mathrm{C}$ for $30 \mathrm{sec}$. To analyze the qPCR data, relative quantification was performed using the $2^{-\Delta \Delta \mathrm{Cq}}$ method with human GAPDH as the internal control gene; data are expressed as relative fold-changes (32). The sequences of human SHP and GAPDH primers were as follows: SHP forward, 5'-TCCTCTTCAACC CCGATGTG-3' and reverse, 5'-CAGGGTTCCAGGACTTCA CAC-3': GAPDH forward, 5'-TCGGAGTCAACGGATTTG GT-3' and reverse, 5'-TTCCCGTTCTCAGCCTTGAC-3'.

Statistical analysis. The associations between clinicopathological or hematological factors and SHP levels were analyzed using Pearson's $\chi^{2}$ test and Fisher's exact test, and survival curves were created by the Kaplan-Meier method. The prognostic value of SHP expression was evaluated using the log-rank test for univariate analysis and the Cox proportional hazards model for multivariate analyses. A backward stepwise selection of covariates was used for the Cox proportional hazards model, and $\mathrm{P}<0.1$ was defined as the threshold for covariate inclusion. $\mathrm{P}<0.05$ was considered to indicate a statistically significant difference. All statistical analyses were conducted using PASW statistical software (version 17.0; SPSS, Inc.).

\section{Results}

Bioinformatics analysis of SHP expression in READ. Numerous studies have shown that SHP prevents or controls acute inflammatory responses in innate immune cells (24-27). These studies have led to the hypothesis that SHP may play a role in the regulation of cancer-associated inflammation. If this hypothesis is correct, a low SHP expression should inhibit an adequate immune response in READ. To test this, GSEA was performed by comparing high and low SHP mRNA expression groups in hallmark gene sets using TCGA mRNA-Seq data (Fig. 1A). GSEA revealed a significant difference (FDR, $<0.25$; nominal $\mathrm{P}<0.05$ ) between the two groups with regard to enrichment with genes from the MSigDB Collection (h.all. v6.2.symbols.gmt), with details shown in Fig. 1A. Moreover, Fig. 1A shows that inflammatory responses [normalized enrichment score (NES), -1.67507], NOTCH signaling (NES, -1.70074), IL2-STAT5 signaling (NES, -1.58612) and KRAS signaling (NES, -1.5011) were differentially enriched in the SHP low expression group.

The GSEA results suggest that several pathways associated with cancer progression were upregulated in patients with READ who had low SHP expression. Therefore, the OncoLnc database was used to investigate whether the mRNA expression level of SHP was a prognostic factor for READ. The data from the OncoLnc database, which stores gene expression data and clinical information for READ, were analyzed. The results revealed that patients with READ who had low SHP mRNA expression levels had a worse prognosis than those with high SHP mRNA levels ( $\mathrm{P}=0.0319$; Fig. 1B).

Association between SHP expression and patient characteristics. Since GSEA and survival analysis rely on the expression profile of mRNA, the SHP protein level was examined in clinical samples derived from patients with LARC treated with pRCT. IHC showed that SHP was expressed mainly in the nuclei but also in the cytoplasm. Representative SHP staining results are shown in Fig. 2. A high level of SHP expression was detected in the tumor tissues of $37 / 89$ patients with READ (41.6\%), whereas 52 patients were categorized into the low expression group (Table I).

The clinicopathological characteristics of the 89 patients with LARC according to SHP expression are presented in Table I. The median age of the patients was 62 years (range, 33-81 years). Low SHP expression was positively associated with clinical $\mathrm{N}(\mathrm{cN})$ stage [N (-) vs. N (+); P=0.004]. No significant association was identified between SHP expression and other clinicopathological variables, including age, sex, tumor distance from the anal verge and clinical T (cT) stage.

Since the aforementioned GSEA results indicate that SHP expression is negatively associated with inflammatory responses, the associations between SHP protein expression and systemic inflammatory markers were determined. The associations between SHP expression and hematological characteristics of inflammation in the patients are presented in Table II. Among the various inflammatory markers, low SHP expression was associated with the following hematological parameters: Neutrophil counts $(\mathrm{P}=0.023)$, lymphocyte counts $(\mathrm{P}=0.024)$ and NLR values $(\mathrm{P}=0.023)$. Similarly, patients with low SHP expression tended to present with more highly elevated erythrocyte sedimentation rates (ESRs) than those with high SHP expression (Fig. S1). However, no association was detected between SHP and either platelet counts or PLR values.

Association of SHP expression with pCR to RCT. Next, the relationship between treatment response and patient characteristics was determined using Fisher's exact tests. Following pRCT, a pCR was observed in 19 patients $(21.3 \%)$. The associations between $\mathrm{pCR}$ and patient characteristics, including SHP 
A

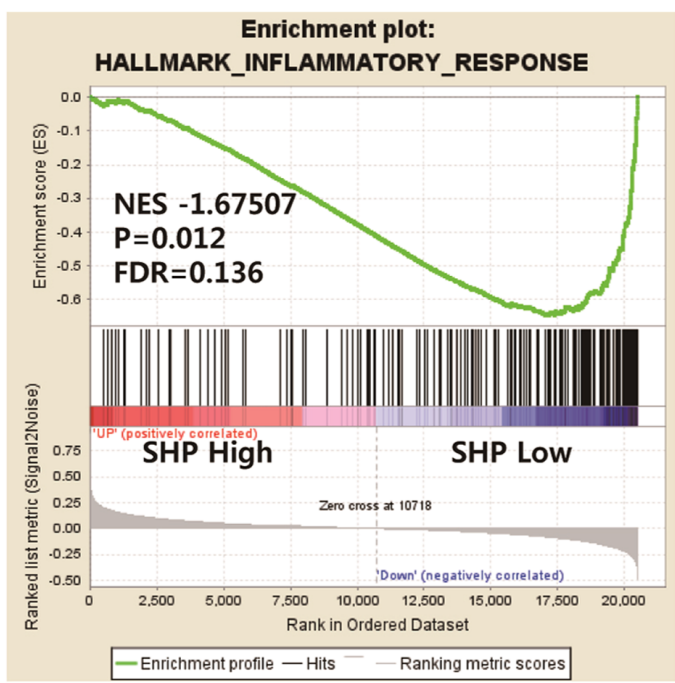

Enrichment plot: HALLMARK_IL2_STAT5_SIGNALING

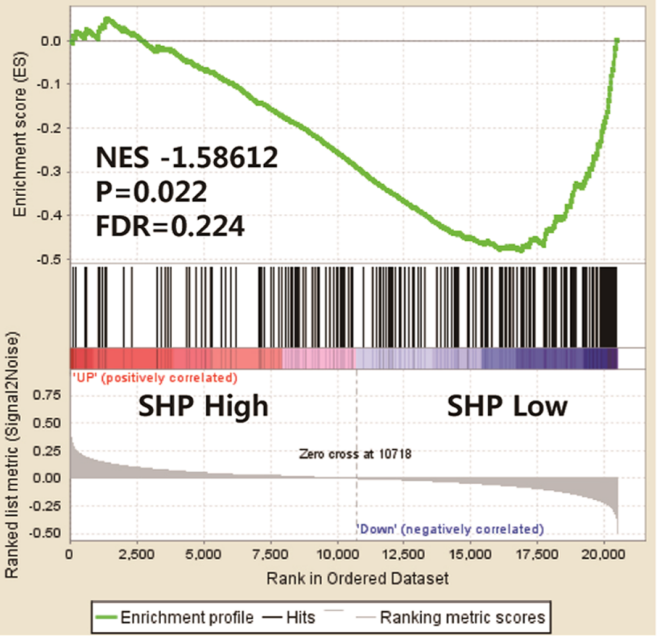

B

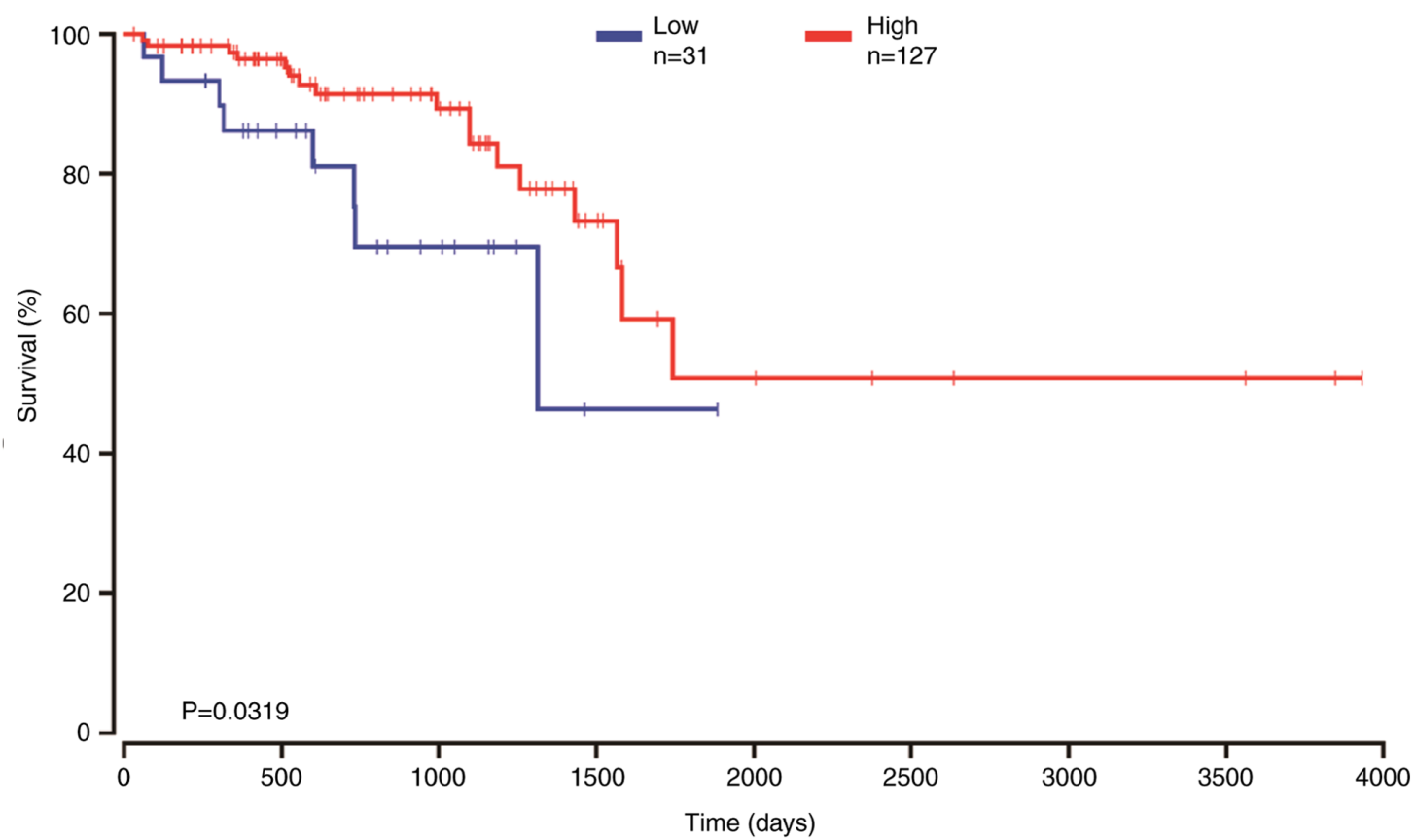

Enrichment plot: HALLMARK_NOTCH_SIGNALING

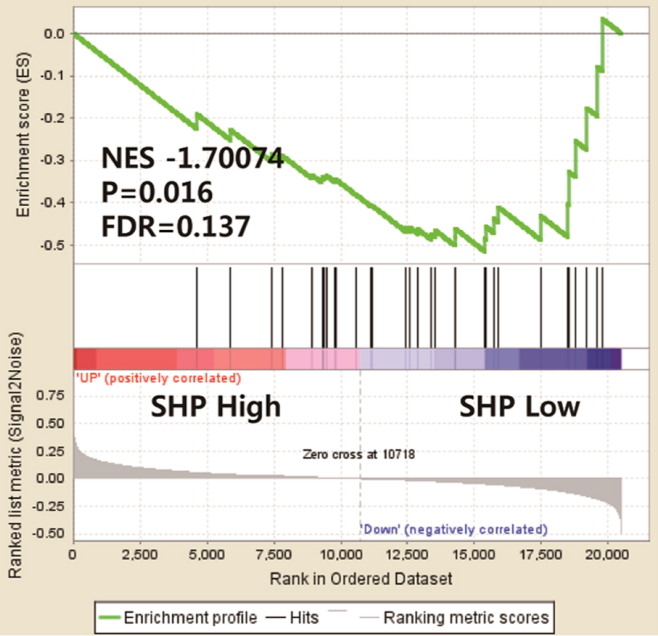

Enrichment plot: HALLMARK_KRAS_SIGNALING_UP
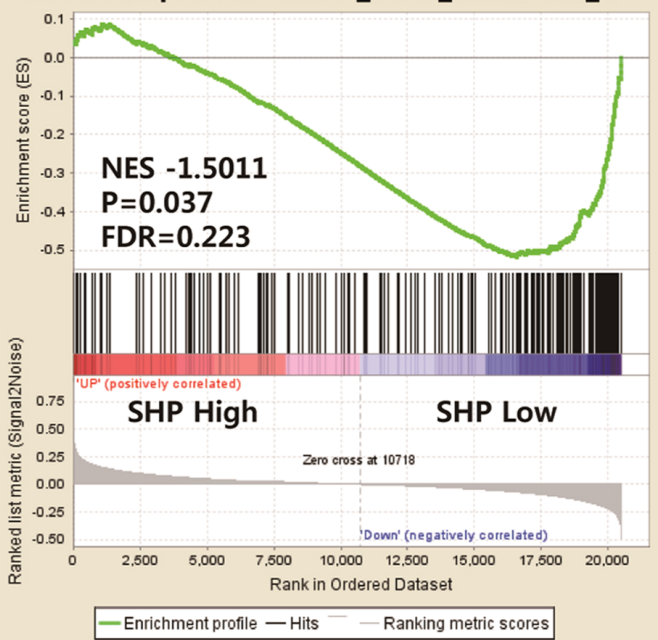

-Enrichment profile - Hits - - Ranking metric scores 
Table II. Association of SHP with hematologic parameters in patients with locally advanced rectal cancer.

\begin{tabular}{|c|c|c|c|c|}
\hline \multirow[b]{2}{*}{ Characteristics } & \multirow[b]{2}{*}{ Total, n (\%) } & \multicolumn{3}{|c|}{ SHP expression, $\mathrm{n}(\%)$} \\
\hline & & Low $(\mathrm{n}=52)$ & High $(n=37)$ & P-value \\
\hline Neutrophil count & & & & 0.023 \\
\hline Below the median & $45(50.6)$ & $21(40.4)$ & $24(64.9)$ & \\
\hline Above the median & $44(49.4)$ & $31(59.6)$ & $13(35.1)$ & \\
\hline Lymphocyte count & & & & 0.024 \\
\hline Below the median & $44(49.4)$ & $23(44.2)$ & $21(56.8)$ & \\
\hline Above the median & $45(50.6)$ & $29(55.8)$ & $16(43.2)$ & \\
\hline Platelet count & & & & 0.761 \\
\hline Below the median & $45(50.6)$ & $27(51.9)$ & $18(48.6)$ & \\
\hline Above the median & $44(49.4)$ & $25(48.1)$ & $19(51.4)$ & \\
\hline NLR & & & & 0.023 \\
\hline Below the median & $45(50.6)$ & $21(40.4)$ & $24(64.9)$ & \\
\hline Above the median & $44(49.4)$ & $31(59.6)$ & $13(35.1)$ & \\
\hline PLR & & & & 0.900 \\
\hline Below the median & $45(50.6)$ & $26(50.0)$ & $19(51.4)$ & \\
\hline Above the median & $44(49.4)$ & $26(50.0)$ & $18(48.6)$ & \\
\hline
\end{tabular}

Data were analyzed using Pearson's $\chi^{2}$ test. SHP, small heterodimer partner; NLR, neutrophil-lymphocyte ratio; PLR, platelet-lymphocyte ratio.
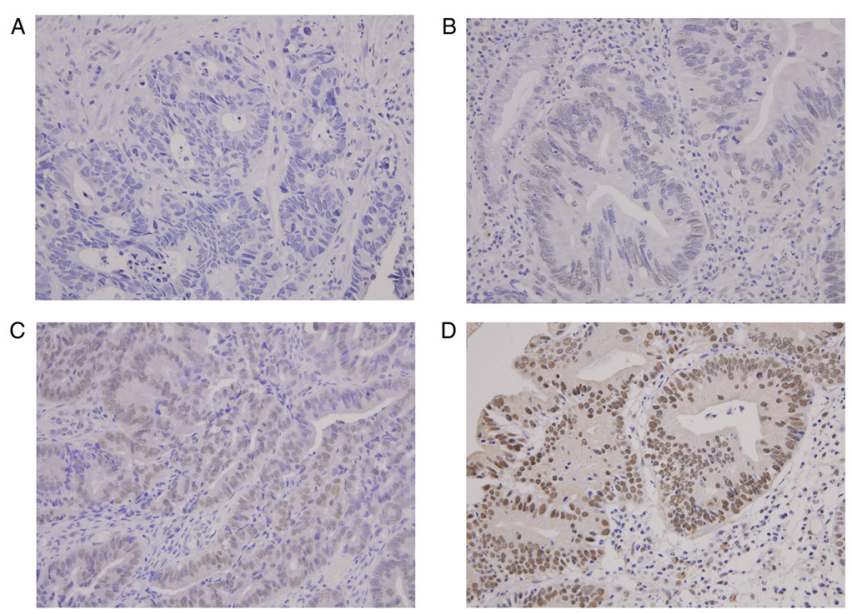

Figure 2. Representative photomicrographs of SHP immunohistochemica staining in human rectal cancer tissues. (A) No staining intensity, (B) weak staining intensity, (C) moderate staining intensity and (D) strong staining intensity (original magnification, $\mathrm{x} 400$ ). SHP, small heterodimer partner.

expression and clinical/hematological factors, are presented in Table III. The nuclear expression of SHP was significantly higher in patients with $\mathrm{pCR}$, as compared with those without (67.6 vs. $32.4 \%$; $\mathrm{P}=0.038$ ). In addition, a significant association was detected between pathologic tumor response and other factors, including the low NLR (66.7 vs. 33.3\%; $\mathrm{P}=0.009)$ and PLR (66.7 vs. 33.3\%; $\mathrm{P}=0.009$ ). No significant association was identified between $\mathrm{pCR}$ and age, sex, tumor distance from the anal verge, cT or cN stage, and neutrophil, lymphocyte and platelet counts.

To validate the relationship between the mRNA expression of SHP and pCR in patients with LARC, RT-qPCR analysis of LARC tissues was conducted. As shown in Fig. S2, the mRNA expression of SHP was significantly higher $(\mathrm{P}=0.0283)$ in patients that achieved a pCR to $\mathrm{pRCT}$, as compared with those in the non-responder group. Additionally, whether the expression level of SHP is affected by pRCT was investigated. To evaluate the change in SHP expression between pretreatment and post-pRCT surgical specimens, a publicly available transcriptome dataset (GSE15781) was analyzed (Fig. S3). A non-significant reduction in the mRNA expression of SHP was observed in residual cancer tissues following pRCT treatment (Fig. S3).

Association between SHP expression and survival. The median follow-up time was 54.0 months (range, 16-88 months) for all patients and 58.5 months (range, 16-88 months) for the surviving patients. Local and distant failure were observed in $13(14.6 \%)$ and $24(27.0 \%)$ cases, respectively. The 5-year OS and distant metastasis-free survival (DMFS) rates were 81.3 and $73.3 \%$, respectively (Fig. 3A).

Since SHP expression was found to be significantly associated with OS and DMFS, the effects of prognostic factors on the two types of survival were analyzed. Table IV shows the association between potential prognostic factors and OS, as determined by univariate and multivariate analyses. In the univariate analysis, pCR and SHP expression were significantly associated with OS. In addition, the 52 patients with low nuclear SHP expression exhibited poorer OS rates than the 37 patients with high nuclear SHP expression (72.3 vs. 94.4\%; Fig. 3A). Furthermore, the pre-pRCT CEA level and the NLR were found to be marginally significant prognostic factors for OS. Variables with $\mathrm{P}<0.1$, based on univariate analysis, were entered into a Cox proportional hazards model for the multivariate analysis of OS. Multivariate analysis confirmed that pre-pRCT 
Table III. Analysis of predictive factors associated with pathologic complete response.

\begin{tabular}{|c|c|c|c|}
\hline \multirow[b]{3}{*}{ Characteristics } & \multicolumn{3}{|c|}{ Pathologic complete response, n (\%) } \\
\hline & No & Yes & \\
\hline & $70(78.7 \%)$ & $19(21.3)$ & P-value \\
\hline Age, years & & & 0.790 \\
\hline$<60$ & $44(77.2)$ & $13(22.8)$ & \\
\hline$\geq 60$ & $26(81.3)$ & $6(18.7)$ & \\
\hline Sex & & & 0.576 \\
\hline Male & $50(76.9)$ & $15(23.1)$ & \\
\hline Female & $20(83.3)$ & $4(16.7)$ & \\
\hline Tumor distance from anal verge, $\mathrm{cm}$ & & & 0.588 \\
\hline$<6$ & $47(81.0)$ & $11(19.0)$ & \\
\hline$\geq 6$ & $23(83.9)$ & $8(16.1)$ & \\
\hline CEA before RCT, ng/ml & & & 0.429 \\
\hline$\leq 5$ & $44(75.9)$ & $14(24.1)$ & \\
\hline$>5$ & $26(83.9)$ & $5(16.1)$ & \\
\hline cT stage & & & 0.727 \\
\hline $\mathrm{T} 2-3$ & $59(77.6)$ & $17(22.4)$ & \\
\hline $\mathrm{T} 4$ & $11(84.7)$ & $2(15.4)$ & \\
\hline cN stage & & & 1.000 \\
\hline $\mathrm{N}(-)$ & $5(83.3)$ & $1(16.7)$ & \\
\hline $\mathrm{N}(+)$ & $65(78.3)$ & $18(21.7)$ & \\
\hline SHP & & & 0.038 \\
\hline Low & $45(86.5)$ & $7(13.5)$ & \\
\hline High & $25(67.6)$ & $12(32.4)$ & \\
\hline Neutrophil count & & & 0.606 \\
\hline Below the median & $34(75.6)$ & $11(24.4)$ & \\
\hline Above the median & $36(81.8)$ & $8(18.2)$ & \\
\hline Lymphocyte count & & & 0.302 \\
\hline Below the median & $37(84.1)$ & $7(15.9)$ & \\
\hline Above the median & $33(73.3)$ & $12(26.7)$ & \\
\hline Platelet count & & & 0.606 \\
\hline Below the median & $34(75.6)$ & $11(24.4)$ & \\
\hline Above the median & $36(81.8)$ & $8(18.2)$ & \\
\hline NLR & & & 0.009 \\
\hline Below the median & $30(66.7)$ & $15(33.3)$ & \\
\hline Above the median & $40(90.9)$ & $4(9.1)$ & \\
\hline PLR & & & 0.009 \\
\hline Below the median & $30(66.7)$ & $15(33.3)$ & \\
\hline Above the median & $40(90.9)$ & $4(9.1)$ & \\
\hline
\end{tabular}

Data were analyzed using Fisher's exact test. CEA, carcinoembryonic antigen; RCT, radiochemotherapy; cT, clinical T; cN, clinical N; SHP, small heterodimer partner; NLR, neutrophil-lymphocyte ratio; PLR, platelet-lymphocyte ratio.

SHP and CEA levels were independent prognostic factors for OS at a marginal level of significance. Several parameters were found to be associated with DMFS (Table V). In addition, 52 patients with low nuclear SHP expression exhibited poorer DMFS rates compared with the 37 patients with high nuclear SHP expression (58.5 vs. $89.0 \%$; Fig. 3B). In the univariate analysis, pCR and SHP expression were significant prognostic factors for DMFS. In the multivariate analysis, SHP expression remained significant (hazard ratio, 0.315; 95\% confidence interval, 0.107-0.932; $\mathrm{P}=0.037$ ).

As Table II shows, significant associations were identified between SHP protein expression and various hematological 

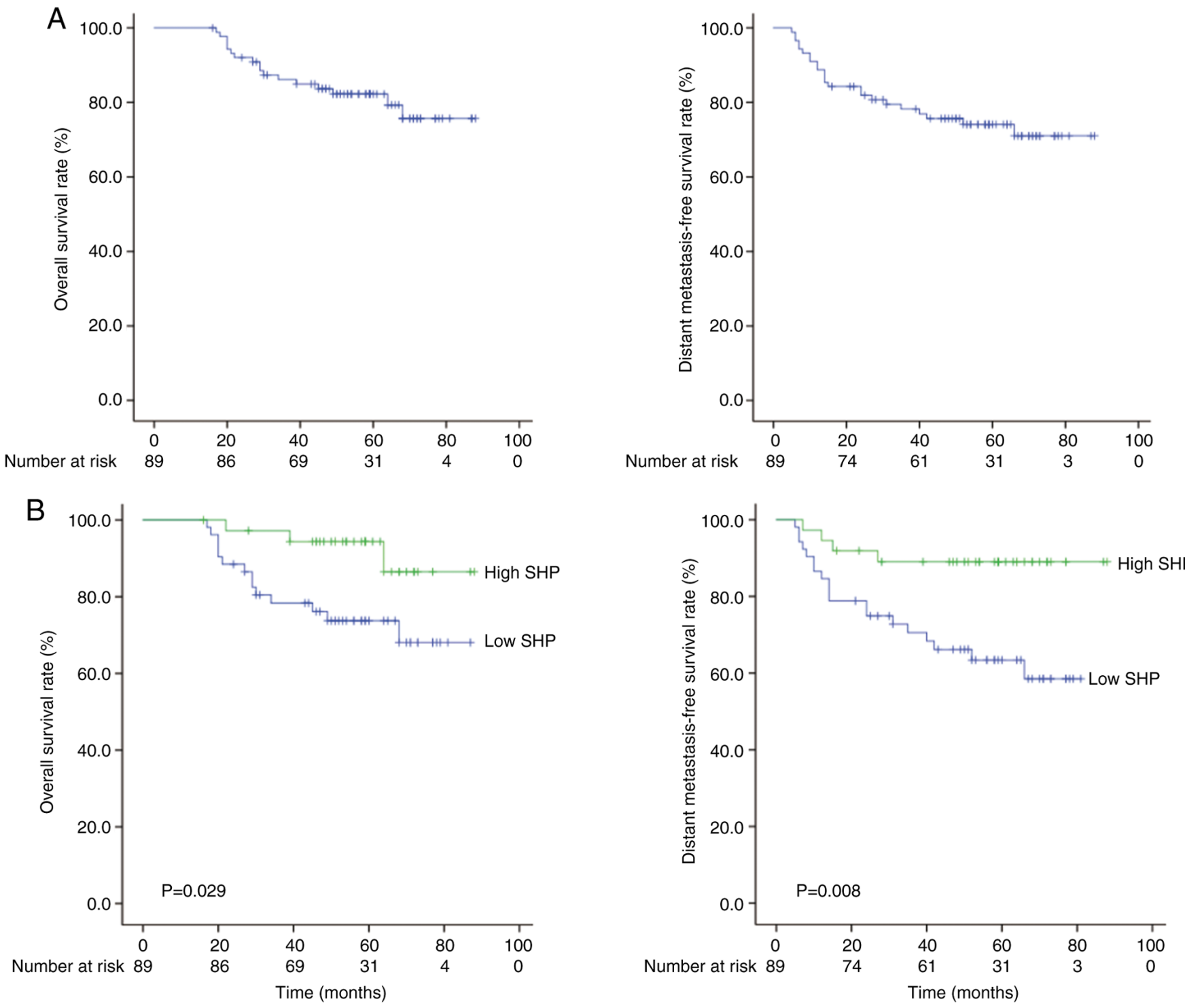

Figure 3. Survival curves for patients with LARC according to SHP expression. (A) OS and DMFS for the entire patient cohort. (B) OS and DMFS curves for patients with LARC, according to SHP expression. Blue lines represent the patients with SHP low expression LARC ( $\mathrm{n}=52$ ) and green lines represent those with SHP high expression $(n=37)$. Patients with low SHP expression had a shorter OS and DMFS than those with high SHP expression. LARC, advanced rectal cancer; SHP, small heterodimer partner; OS, overall survival; DMFS, distant metastasis-free survival.

parameters. Among the hematological parameters associated with SHP, the NLR was a predictor of pCR and OS. Therefore, the 89 cases of READ were classified into three groups, according to the SHP expression and NLR: Group 1, high SHP expression and low NLR ( $\mathrm{n}=24)$; group 2, high SHP expression and high NLR or low SHP expression and low NLR ( $n=34)$; group 3, low SHP and high NLR ( $=31$ ). Kaplan-Meier analysis (Fig. 4) indicated that patients with a high NLR and low SHP expression had the shortest OS and DMSF, and patients with a low NLR and a high SHP expression had the longest OS and DMFS ( $\mathrm{P}=0.009$ and $\mathrm{P}=0.021$, respectively; Fig. 4).

Correlation of SHP expression with immune cell infiltration and programmed death-ligand $1(P D-L 1)$. To investigate the relationship between SHP and diverse immune infiltrating cells, the correlations between SHP and immune marker sets of various READ immune cells in the TIMER databases were determined. Notably, neutrophil $(\mathrm{r}=-0.274 ; \mathrm{P}=0.00895)$ and
$\mathrm{CD}^{+} \mathrm{T}$ cell $(\mathrm{r}=-0.221 ; \mathrm{P}=0.0363)$ infiltration were negatively correlated with SHP in patients with READ (Fig. 5A). Generally, $\mathrm{CD} 8^{+} \mathrm{T}$-cell infiltration is a favorable prognostic factor in patients with READ (33). However, patients with high PD-L1 expression levels and $\mathrm{CD}^{+} \mathrm{T}$ cell densities have been reported to exhibit impaired progression-free survival and OS (34). Therefore, the relationship between SHP expression and the gene expression of immune-suppressive molecules, including PD-L1 (also known as CD274), indoleamine 2,3-dioxygenase 1 and cytotoxic T-lymphocyte associated protein 4 , was examined in patients with READ. Of the various molecules tested, a negative correlation was observed between SHP and PD-L1 ( $\mathrm{r}=-0.204, \mathrm{P}=0.00837$; Fig. 5B).

\section{Discussion}

In the present study, a data-driven approach was used to investigate the role of SHP in READ using GSEA. The 
Table IV. Prognostic factor analysis for overall survival.

P-value

Prognostic factor

5-year overall survival rate $(\%)$

Univariate analysis

Multivariate analysis

Age, years

$<60$

77.8

$\geq 60$

90.4

Sex

Male

82.3

Female

81.5

Tumor distance from anal verge, $\mathrm{cm}$

$<6$

83.3

$\geq 6$

80.3

CEA before CRT, ng/ml

0.093

$\leq 5$

87.3

$>5$

72.8

cT stage

T2-3

82.0

T4

80.9

$\mathrm{cN}$ stage

$\mathrm{N}(-)$

100.0

$\mathrm{N}(+)$

79.9

Pathologic complete response

No

77.4

Yes

100

0.029

Low

73.7

High

94.4

Neutrophil count

Below the median

83.3

Above the median

81.2

Lymphocyte count

Below the median

81.6

Above the median

82.6

NLR

Below the median

87.4

77.1

Above the median

79.0

Below the median

85.6

Above the median

PLR

80.8

Below the median

83.5

Above the median

CEA, carcinoembryonic antigen; RCT, radiochemotherapy; cT, clinical T; cN, clinical N; SHP, small heterodimer partner; NLR, neutrophil-lymphocyte ratio; PLR, platelet-lymphocyte ratio.

results revealed the following information about the potential underlying mechanism: A low SHP mRNA expression in READ was associated with inflammatory responses, as well as NOTCH, IL2-STAT5 and KRAS signalling. These signaling pathways are associated with treatment resistance and poor prognosis (35-38). The clinical significance of SHP expression in READ was also examined using the OncoLnc database. Kaplan-Meier survival curves demonstrated that a low SHP mRNA expression is a poor prognostic factor in READ. Next, the prognostic significance of SHP expression 
Table V. Prognostic factor analysis for distant metastasis-free survival.

\begin{tabular}{|c|c|c|c|}
\hline \multirow[b]{2}{*}{ Prognostic factor } & \multirow{2}{*}{$\begin{array}{c}\text { 5-year distant } \\
\text { metastasis-free survival rate }(\%)\end{array}$} & \multicolumn{2}{|c|}{ P-value } \\
\hline & & Univariate analysis & Multivariate analysis \\
\hline Age, years & & 0.257 & \\
\hline$<60$ & 70.6 & & \\
\hline$\geq 60$ & 80.4 & & \\
\hline Sex & & 0.950 & \\
\hline Male & 74.8 & & \\
\hline Female & 70.8 & & \\
\hline Tumor distance from anal verge, $\mathrm{cm}$ & & 0.355 & \\
\hline$<6$ & 76.9 & & \\
\hline$\geq 6$ & 69.2 & & \\
\hline CEA before CRT, ng/ml & & 0.438 & \\
\hline$\leq 5$ & 76.5 & & \\
\hline$>5$ & 69.9 & & \\
\hline cT stage & & 0.821 & \\
\hline $\mathrm{T} 2-3$ & 72.3 & & \\
\hline $\mathrm{T} 4$ & 70.5 & & \\
\hline cN stage & & 0.542 & \\
\hline $\mathrm{N}(-)$ & 83.3 & & \\
\hline $\mathrm{N}(+)$ & 73.3 & & \\
\hline Pathologic complete response & & 0.024 & 0.099 \\
\hline No & 68.3 & & \\
\hline Yes & 94.7 & & \\
\hline SHP & & 0.008 & 0.037 \\
\hline Low & 63.4 & & \\
\hline High & 89.0 & & \\
\hline Neutrophil count & & 0.661 & \\
\hline Below the median & 71.9 & & \\
\hline Above the median & 75.8 & & \\
\hline Lymphocyte count & & 0.821 & \\
\hline Below the median & 74.1 & & \\
\hline Above the median & 74.1 & & \\
\hline NLR & & 0.348 & \\
\hline Below the median & 78.5 & & \\
\hline Above the median & 69.3 & & \\
\hline Platelet count & & 0.735 & \\
\hline Below the median & 75.6 & & \\
\hline Above the median & 72.3 & & \\
\hline PLR & & 0.794 & \\
\hline Below the median & 76.8 & & \\
\hline Above the median & 71.7 & & \\
\hline
\end{tabular}

CEA, carcinoembryonic antigen; RCT, radiochemotherapy; cT, clinical T; cN, clinical N; SHP, small heterodimer partner; NLR, neutrophil-lymphocyte ratio; PLR, platelet-lymphocyte ratio.

was investigated in LARC using IHC data from Chungnam National University Hospital. Analysis of the IHC and clinical data from this hospital confirmed the results obtained from public databases, suggesting that SHP protein expression is a favorable prognostic factor in LARC. Furthermore, the present results showed that the mRNA and protein expression of SHP is associated with pCR and DMFS in pRCT-treated LARC patients. These findings suggest that the nuclear expression 

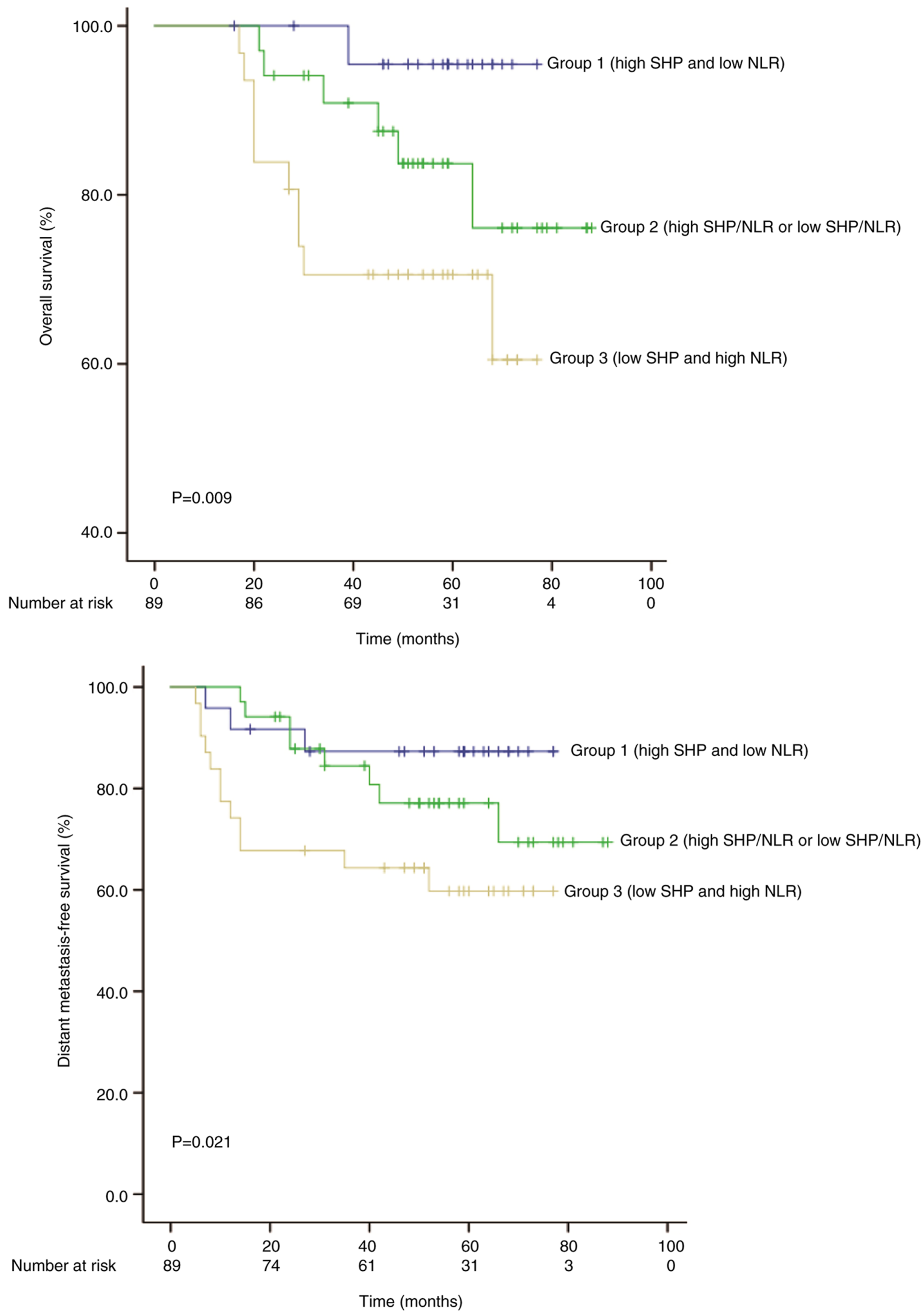

Figure 4. Combination of SHP expression and NLR as a prognostic marker for LARC following pRCT. Overall survival outcomes for LARC following pCRT stratified by SHP expression and NLR. Group 1, high SHP and low NLR (n=24); Group 2, high SHP/NLR or low SHP/NLR (n=34); Group 3, low SHP and high NLR ( $n=31)$. Patients with low SHP expression and high NLR had the poorest overall and distant metastasis-free survival times among the three groups. SHP, small heterodimer partner; NLR, neutrophil-lymphocyte ratio; LARC, locally advanced rectal cancer; pRCT, preoperative radiochemotherapy.

of SHP may be used as an indicator of favorable prognosis for LARC patients who receive pRCT. To date, no clinical studies have evaluated the prognostic role of SHP in LARC. To the best of our knowledge, this study is the first to show 

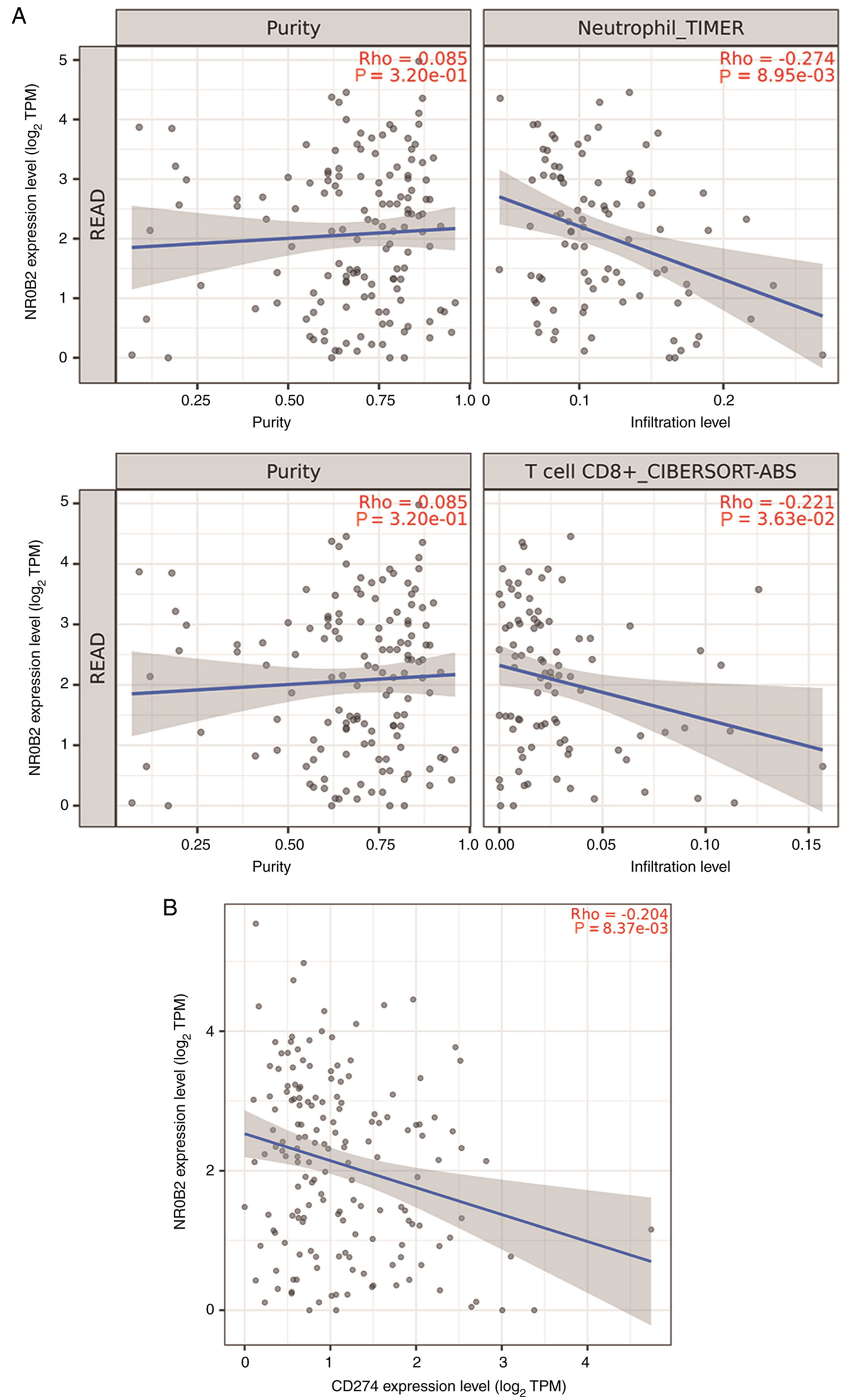

Figure 5. Correlation between SHP (NR0B2) mRNA expression and tumor immunity in READ. (A) Negative correlation of SHP expression with neutrophils and CD8 ${ }^{+} \mathrm{T}$ cells in READ. (B) Negative correlation between SHP expression and PD-L1. SHP, small heterodimer partner; READ, rectal adenocarcinoma; PD-L1, programmed death-ligand 1; TPM, transcripts per million. 
the predictive and prognostic significance of SHP expression in LARC.

Cancer-related local and systemic inflammation has been identified as a key player in tumor invasion and metastasis $(23,35,39)$. It has been demonstrated that several biomarkers and hematological indices are representative of the local immune response, including tumor necrosis factor, inflammasomes, cytokines, chemokines and transcription factors, as well as systemic inflammatory markers, such as acute-phase proteins and circulating immune cells $(23,40)$. Among these, the NLR, PLR, LMR, albumin, ESR and CRP levels in patients with cancer are common prognostic factors, due to their ease of use in clinical practice $(23,40)$. In colorectal cancer, the NLR and PLR are associated with pCR or primary tumor downstaging following pRCT (41-44). Similarly, the results of the present study revealed that hematological parameters, including the NLR and PLR, are significant predictors of $\mathrm{pCR}$ following $\mathrm{pRCT}$ for rectal cancer. Due to the detrimental effect of cancer-related inflammation on the response to radiotherapy, there is substantial interest in therapeutic strategies for manipulating the inflammatory response. Thus, there is a growing interest in novel approaches for targeting cancer-related inflammatory pathways in combination with radiation therapy. A large variety of natural and synthetic compounds have been reported to interfere with cancer-related inflammation through the regulation of various molecular pathways, including NF- $\mathrm{B}, \mathrm{STAT} 3$, hypoxia-inducible factor 1 and prostaglandin-endoperoxide synthase 2 pathways, and are regarded as putative radiosensitizing agents (45).

Our previous studies have demonstrated the importance of SHP in the regulation of innate immune and inflammatory responses against pathogen invasion (24-27). However, no study has yet evaluated the role of SHP in cancer immunology. In the present study, GSEA revealed that inflammatory responses are differentially enriched in patients with READ and low SHP expression. Furthermore, a negative association was identified between SHP expression and the NLR. A previous study reported a close association of immune suppression with an increased NLR (46). These results suggest that low SHP expression may generate an immunosuppressive tumor microenvironment by promoting cancer-associated inflammation. Another previous study reported that patients with gastric or gastro-esophageal junction adenocarcinomas who have a high PD-L1 expression and high $\mathrm{CD}^{+} \mathrm{T}$ cell density have poor survival, suggesting a potentially adaptive immune resistance mechanism (34). In this situation, cancer cells frequently escape immune cell recognition via the upregulation of immune checkpoint molecules, such as PD-1/PD-L1 and cytotoxic T-lymphocyte antigen $4(47,48)$. Similarly, despite the negative association between SHP expression and $\mathrm{CD}^{+} \mathrm{T}$ cell infiltration, the present study observed that patients with low SHP expression had a poor prognosis. Among the various molecules studied, a negative association was identified between SHP and PD-L1. Collectively, these results suggest that low SHP expression is strongly associated with immune-suppressive functions via the upregulation of PD-L1, resulting in poor prognosis in LARC.

It is important to acknowledge the methodological limitations of the present retrospective study. First, it included a small number of patients from a single center. Furthermore, 46 LARC cases from the study period were excluded due to the unavailability of paraffin blocks and only 89 LARC cases were evaluated for SHP IHC status. Thus, the analysis may have been subject to potential selection bias. Despite its limitations, the study is of value because the primary data from the patients were validated by various bioinformatics approaches.

In conclusion, the present pilot study assessed the role of SHP expression in rectal cancer. The IHC results suggested that the expression level of SHP is associated with systemic inflammation, treatment outcomes and prognosis in LARC. The bioinformatics analysis results support the observation that low SHP expression is associated with cancer-related inflammation, immunosuppression and poor prognosis. Therefore, these findings provide valuable insights for the identification of potential therapeutic targets and promising prognostic markers in rectal cancer.

\section{Acknowledgements}

The authors would like to thank Professor Hueng-Sik Choi (School of Biological Sciences and Technology, Chonnam National University) for critical reading of the manuscript.

\section{Funding}

This study was supported by National Research Foundation of Korea grants funded by the Korean Government (Ministry of Science, ICT and Future Planning; grant nos. 2017R1A5A2015385 and NRF-2019M3E5D1A02068546) and by a grant from 2019 from Chungnam National University.

\section{Availability of data and materials}

The datasets used and/or analyzed during the current study are available from the corresponding author on reasonable request.

\section{Authors' contributions}

SK took part in conception and design of the study and the acquisition, analysis and interpretation of data, and drafted the manuscript. EKJ and JMK took part in conception and design of the study, critical revision of the manuscript for important intellectual content and supervision of the study. SK, MJ, MKY, MJC and JSK took part in the acquisition of data and supervision. SK, EKJ and JMK confirmed the authenticity of all the raw data. All authors read and approved the final manuscript.

\section{Ethics approval and consent to participate}

The current study was a retrospective study with approval from the institutional review board of Chungnam National University Hospital (IRB no. 2017-07-037) and the contents of this study are open to the public at the hospital. All methods were performed in accordance with the 1975 Declaration of Helsinki. All patients signed an informed consent form.

\section{Patient consent for publication}

Not applicable. 


\section{Competing interests}

The authors declare that they have no competing interests.

\section{References}

1. Kuo LJ, Liu MC, Jian JJ, Horng CF, Cheng TI, Chen CM, Fang WT and Chung YL: Is final TNM staging a predictor for survival in locally advanced rectal cancer after preoperative chemoradiation therapy? Ann Surg Oncol 14: 2766-2772, 2007.

2. Seol W, Choi HS and Moore DD: An orphan nuclear hormone receptor that lacks a DNA binding domain and heterodimerizes with other receptors. Science 272: 1336-1339, 1996.

3. Seol W, Chung M and Moore DD: Novel receptor interaction and repression domains in the orphan receptor SHP. Mol Cell Biol 17: 7126-7131, 1997

4. Burris TP, Solt LA, Wang Y, Crumbley C, Banerjee S, Griffett K Lundasen T, Hughes T and Kojetin DJ: Nuclear receptors and their selective pharmacologic modulators. Pharmacol Rev 65: 710-778, 2013.

5. Kim MK, Chanda D, Lee IK, Choi HS and Park KG: Targeting orphan nuclear receptor SHP in the treatment of metabolic diseases. Expert Opin Ther Targets 14: 453-466, 2010.

6. Zhang Y, Hagedorn $\mathrm{CH}$ and Wang L: Role of nuclear receptor SHP in metabolism and cancer. Biochim Biophys Acta 1812: 893-908, 2011.

7. Zhang Y, Soto J, Park K, Viswanath G, Kuwada S, Abel ED and Wang L: Nuclear receptor SHP, a death receptor that targets mitochondria, induces apoptosis and inhibits tumor growth. Mol Cell Biol 30: 1341-1356, 2010.

8. Zhang Y, Xu P, Park K, Choi Y, Moore DD and Wang L: Orphan receptor small heterodimer partner suppresses tumorigenesis by modulating cyclin D1 expression and cellular proliferation. Hepatology 48: 289-298, 2008.

9. He N, Park K, Zhang Y, Huang J, Lu S and Wang L: Epigenetic inhibition of nuclear receptor small heterodimer partner is associated with and regulates hepatocellular carcinoma growth Gastroenterology 134: 793-802, 2008

10. Song G, Zhang Y and Wang L: MicroRNA-206 targets notch3, activates apoptosis, and inhibits tumor cell migration and focus formation. J Biol Chem 284: 31921-31927, 2009.

11. Seol W, Hanstein B, Brown M and Moore DD: Inhibition of estrogen receptor action by the orphan receptor SHP (short heterodimer partner). Mol Endocrinol 12: 1551-1557, 1998

12. Johansson L, Thomsen JS, Damdimopoulos AE, Spyrou G Gustafsson JA and Treuter E: The orphan nuclear receptor SHP inhibits agonist-dependent transcriptional activity of estrogen receptors ERalpha and ERbeta. J Biol Chem 274: 345-353, 1999.

13. Lai K, Harnish DC and Evans MJ: Estrogen receptor alpha regulates expression of the orphan receptor small heterodimer partner. J Biol Chem 278: 36418-36429, 2003.

14. Nishizawa H, Yamagata K, Shimomura I, Takahashi M, Kuriyama H, Kishida K, Hotta K, Nagaretani H, Maeda N, Matsuda M, et al: Small heterodimer partner, an orphan nuclear receptor, augments peroxisome proliferator-activated receptor gamma transactivation. J Biol Chem 277: 1586-1592, 2002.

15. Rubin GL, Duong JH, Clyne CD, Speed CJ, Murata Y Gong $\mathrm{C}$ and Simpson ER: Ligands for the peroxisomal proliferator-activated receptor gamma and the retinoid $\mathrm{X}$ receptor inhibit aromatase cytochrome P450 (CYP19) expression mediated by promoter II in human breast adipose. Endocrinology 143: 2863-2871, 2002.

16. Swales KE, Korbonits M, Carpenter R, Walsh DT, Warner TD and Bishop-Bailey D: The farnesoid X receptor is expressed in breast cancer and regulates apoptosis and aromatase expression. Cancer Res 66: 10120-10126, 2006.

17. Coussens LM and Werb Z: Inflammation and cancer. Nature 420 860-867, 2002

18. Woo HD, Kim K and Kim J: Association between preoperative C-reactive protein level and colorectal cancer survival: A meta-analysis. Cancer Causes Control 26: 1661-1670, 2015.

19. Choi N, Kim JH, Chie EK, Gim J and Kang HC: A meta-analysis of the impact of neutrophil-to-lymphocyte ratio on treatment outcomes after radiotherapy for solid tumors. Medicine (Baltimore) 98: e15369, 2019.

20. Song W, Wang K, Zhang RJ and Zou SB: Prognostic value of the lymphocyte monocyte ratio in patients with colorectal cancer: A meta-analysis. Medicine (Baltimore) 95: e5540, 2016.
21. Tan D, Fu Y, Su Q and Wang H: Prognostic role of platelet-lymphocyte ratio in colorectal cancer: A systematic review and meta-analysis. Medicine (Baltimore) 95: e3837, 2016.

22. Park JH, Richards CH, McMillan DC, Horgan PG and Roxburgh CSD: The relationship between tumour stroma percentage, the tumour microenvironment and survival in patients with primary operable colorectal cancer. Ann Oncol 25 644-651, 2014.

23. Diakos CI, Charles KA, McMillan DC and Clarke SJ: Cancer-related inflammation and treatment effectiveness. Lancet Oncol 15: e493-e503, 2014.

24. Yuk JM, Jin HS and Jo EK: Small heterodimer partner and innate immune regulation. Endocrinol Metab (Seoul) 31: 17-24, 2016.

25. Yuk JM, Shin DM, Lee HM, Kim JJ, Kim SW, Jin HS, Yang CS, Park KA, Chanda D, Kim DK, et al: The orphan nuclear receptor SHP acts as a negative regulator in inflammatory signaling triggered by Toll-like receptors. Nat Immunol 12: 742-751, 2011.

26. Yang CS, Yuk JM, Kim JJ, Hwang JH, Lee CH, Kim JM, Oh GT, Choi HS and Jo EK: Small heterodimer partner-targeting therapy inhibits systemic inflammatory responses through mitochondrial uncoupling protein 2. PLoS One 8: e63435, 2013.

27. Yang CS, Kim JJ, Kim TS, Lee PY, Kim SY, Lee HM, Shin DM, Nguyen LT, Lee MS, Jin HS, et al: Small heterodimer partner interacts with NLRP3 and negatively regulates activation of the NLRP3 inflammasome. Nat Commun 6: 6115, 2015.

28. Eun HS, Cho SY, Lee BS, Kim S, Song IS, Chun $\mathrm{K}$, Oh $\mathrm{CH}$, Yeo MK, Kim SH and Kim KH: Cytochrome P450 4A11 expression in tumor cells: A favorable prognostic factor for hepatocellular carcinoma patients. J Gastroenterol Hepatol 34: 224-233, 2019

29. Snipstad K, Fenton CG, Kjaeve J, Cui G, Anderssen E and Paulssen RH: New specific molecular targets for radio-chemotherapy of rectal cancer. Mol Oncol 4: 52-64, 2010.

30. Li T, Fan J, Wang B, Traugh N, Chen Q, Liu JS, Li B and Liu XS: TIMER: A web server for comprehensive analysis of tumor-infiltrating immune cells. Cancer Res 77: e108-e110, 2017.

31. Weiser MR: AJCC 8th edition: Colorectal cancer. Ann Surg Oncol 25: 1454-1455, 2018.

32. Livak KJ and Schmittgen TD: Analysis of relative gene expression data using real-time quantitative PCR and the 2(-Delta Delta C(T)) method. Methods 25: 402-408, 2001

33. Jochems $C$ and Schlom J: Tumor-infiltrating immune cells and prognosis: The potential link between conventional cancer therapy and immunity. Exp Biol Med (Maywood) 236: 567-579, 2011.

34. Thompson ED, Zahurak M, Murphy A, Cornish T, Cuka N, Abdelfatah E, Yang S, Duncan M, Ahuja N, Taube JM, et al: Patterns of PD-L1 expression and CD8 T cell infiltration in gastric adenocarcinomas and associated immune stroma. Gut 66: 794-801, 2017.

35. Roxburgh CS and McMillan DC: Cancer and systemic inflammation: Treat the tumour and treat the host. Br J Cancer 110 $1409-1412,2014$

36. Tyagi A, Sharma AK and Damodaran C: A review on notch signaling and colorectal cancer. Cells 9: 1549, 2020.

37. Chen S-Y, Chen S, Feng W, Li Z, Luo Y and Zhu X: A STING-related prognostic score predicts high-risk patients of colorectal cancer and provides insights into immunotherapy. Ann Transl Med 9: 14, 2021.

38. Arrington AK, Heinrich EL, Lee W, Duldulao M, Patel S, Sanchez J, Garcia-Aguilar J and Kim J: Prognostic and predictive roles of KRAS mutation in colorectal cancer. Int J Mol Sci 13: 12153-12168, 2012

39. Grivennikov SI, Greten FR and Karin M: Immunity, inflammation, and cancer. Cell 140: 883-899, 2010.

40. Hannisdal E, Tveit KM, Theodorsen L and Høst H: Host markers and prognosis in recurrent rectal carcinomas treated with radiotherapy. Acta Oncol 33: 415-421, 1994.

41. Xiao B, Peng J, Zhang R, Xu J, Wang Y, Fang Y, Lin J, Pan Z and Wu X: Density of CD8+ lymphocytes in biopsy samples combined with the circulating lymphocyte ratio predicts pathologic complete response to chemoradiotherapy for rectal cancer. Cancer Manag Res 9: 701-708, 2017. 
42. Hodek M, Sirák I, Ferko A, Örhalmi J, Hovorková E, Hadži Nikolov D, Paluska P, Kopecký J, Petera J and Vošmik M: Neoadjuvant chemoradiotherapy of rectal carcinoma: Baseline hematologic parameters influencing outcomes. Strahlenther Onkol 192: 632-640, 2016.

43. Kim IY, You SH and Kim YW: Neutrophil-lymphocyte ratio predicts pathologic tumor response and survival after preoperative chemoradiation for rectal cancer. BMC Surg 14: 94, 2014

44. Lee JH, Song C, Kang SB, Lee HS, Lee KW and Kim JS Predicting pathological complete regression with haematological markers during neoadjuvant chemoradiotherapy for locally advanced rectal cancer. Anticancer Res 38: 6905-6910, 2018.

45. Multhoff $\mathrm{G}$ and Radons J: Radiation, inflammation, and immune responses in cancer. Front Oncol 2: 58, 2012.

46. Gonda K, Shibata M, Sato Y, Washio M, Takeshita H, Shigeta H, Ogura M, Oka S and Sakuramoto S: Elevated neutrophil-to-lymphocyte ratio is associated with nutritional impairment, immune suppression, resistance to $S-1$ plus cisplatin, and poor prognosis in patients with stage IV gastric cancer. Mol Clin Oncol 7: 1073-1078, 2017.
47. Topalian SL, Drake CG and Pardoll DM: Immune checkpoint blockade: A common denominator approach to cancer therapy. Cancer Cell 27: 450-461, 2015.

48. McGranahan N, Furness AJ, Rosenthal R, Ramskov S, Lyngaa R, Saini SK, Jamal-Hanjani M, Wilson GA, Birkbak NJ, Hiley CT, et al: Clonal neoantigens elicit T cell immunoreactivity and sensitivity to immune checkpoint blockade. Science 351: 1463-1469, 2016

(C) $($ ) $\mathrm{T}$ This work is licensed under a Creative Commons Attribution-NonCommercial-NoDerivatives 4.0 International (CC BY-NC-ND 4.0) License. 